\title{
HUBBLE SPACE TELESCOPE STUDIES OF THE DENSE CENTRAL REGIONS OF GLOBULAR CLUSTERS
}

\author{
PURAGRA GUHATHAKURTA \\ Univ. of California, Lick Obs., Santa Cruz, CA 95064, USA \\ BRIAN YANNY \\ Fermi National Accelerator Lab., Batavia, IL 60510, USA \\ DONALD P. SCHNEIDER \\ Dept. of Astronomy \& Astrophysics, Pennsylvania State Univ., \\ University Park, PA 16802, USA \\ AND \\ JOHN N. BAHCALL \\ Inst. for Advanced Study, Princeton, NJ 08540, USA
}

\begin{abstract}
We present results from an ongoing program to probe the dense central parts of Galactic globular clusters using multicolor Hubble Space Telescope images (WF/PC-I and WFPC2). Our sample includes the dense clusters M15, 47 Tuc, M30, NGC 6624, M3 and M13. The two main goals of our program are to measure the shape of stellar density profile in clusters (the slope of the density cusp in post core collapse clusters, in particular) and to understand the nature of evolved stellar populations in very dense regions and their variation as a function of radius. The latter includes studies of blue straggler stars and of the central depletion of bright red giants. Our recent WFPC2 study of M15 is described in detail.
\end{abstract}

\section{Introduction}

Many of the highlights of this symposium were provided by recent observations with the Hubble Space Telescope (HST). The high angular resolution and faint stellar detection threshold of HST make it an ideal instrument for the study of dense star clusters. It is instructive to quantify the benefits of high angular resolution in the context of the central region of a dense globular cluster (such as 47 Tuc or M15). The $1^{\prime \prime}$ seeing (FWHM) that is typical 


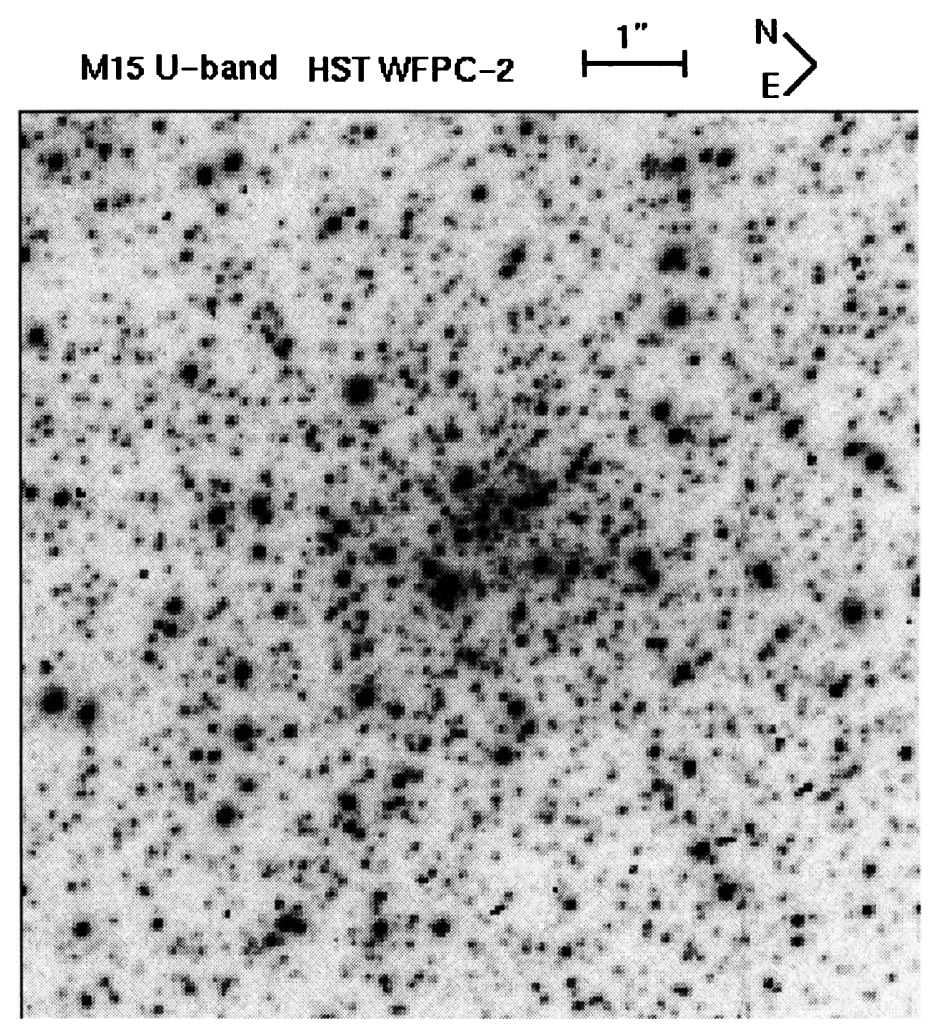

Figure 1. Short $U$ band HST WFPC2 exposure of the central $9^{\prime \prime} \times 9^{\prime \prime}$ of M15. The surface density of stars rises towards the center all the way in to subarcsecond scales. The 0 " 1 resolution of images like the one displayed makes it possible to resolve individual stars down to the main sequence turnoff even near the dense cluster center.

of groundbased observatories causes the blurred images of the brightest stars, those on the upper red giant branch (RGB), to crowd the central region, limiting studies to stars brighter than the horizontal branch (Aurière and Cordoni 1981). The "dynamic range" of such observations-roughly defined to be the range of stellar brightnesses over which accurate photometry is possible-is limited to about 2.5 mag or a factor of 10 . Under very good seeing conditions (FWHM $\sim 0 " .5$ ), it is possible to study subgiants that are up to 5 mag fainter than the tip of the RGB, corresponding to a dynamic range of 100 (Bolte et al. 1993; Stetson 1994). The sharp 0 ". 1 core of the pre-repair HST point spread function represented a great leap forward in our ability to perform complete star identifications down to faint limiting magnitudes, but its broad and complicated wings $\left(r_{\mathrm{PSF}} \sim 2.5\right)$ 
made accurate photometry difficult, if not impossible. The dynamic range of short pre-repair HST exposures of crowded clusters is about 200, barely reaching down to the main sequence turnoff (Lauer et al. 1991; Paresce et al. 1991; Guhathakurta et al. 1992; Sosin and King 1995). This subject has received a tremendous boost thanks to post-repair HST imaging (WFPC2 and FOC+COSTAR) with its dynamic range of over 1000, enabling crowded field photometry of stars up to 2 mag below the turnoff even with exposure times $\lesssim 1 \mathrm{~min}$ (cf. Yanny et al. 1994b; Fig. 1).

Over the last 5 years, we have analyzed HST snapshots of the central regions of several nearby dense Galactic globular clusters. This includes prerepair WF/PC-I $V$ and $I$ images of 47 Tuc (Guhathakurta et al. 1992), M15 (Yanny et al. 1994a), M3 (Guhathakurta et al. 1994), and M13 (Cohen et al. 1996), as well as post-repair WFPC2 $U, B$, and $V$ images of M30 (Yanny et al. 1994b; Guhathakurta et al. 1996a), M15 (Guhathakurta et al. 1996b), and NGC 6624 (Yanny et al. 1996). This paper is based primarily on these datasets.

There are two main themes in our HST studies of globular clusters. The first is the characterization of the stellar surface density profile on subarcsecond scales (slopes, core radii). This paper focusses on new WFPC2 results on M15 (Sec. 2). The broader goal is to place empirical constraints on the physical nature of gravothermal collapse and on the shapes of stellar concentrations around massive black holes. The second theme involves studying the mix of stellar populations as a function of radius (and hence of ambient stellar density) in dense cluster cores, in order to investigate the effects of a dense environment on the evolution of individual stars and the effects of mass segregation. This includes ongoing work on centrally concentrated blue straggler stars (BSSs) in 47 Tuc, M30, and M15 and the depletion of extreme RGB stars near the center of M15 and other post core collapse clusters (Sec. 3).

\section{Density Distribution}

Visible stars in globular clusters are a convenient tracer of the underlying mass distribution. The shape of the stellar luminosity function is such that bright RGB stars contribute a significant fraction of the total cluster light while constituting a very small fraction of the total mass. For example, RGB stars brighter than the horizontal branch represent less than $10 \%$ of all post main sequence stars by number, but are responsible for about two-thirds of the light. Evolved stars in globular clusters have roughly the same mass $\left(M_{\mathrm{TO}}\right)$ despite the fact that their luminosities span a range of nearly $7 \mathrm{mag}$ (factor of 500). For this reason, the number-weighted stellar density (counts) provides a more reliable measure of the mass density than 


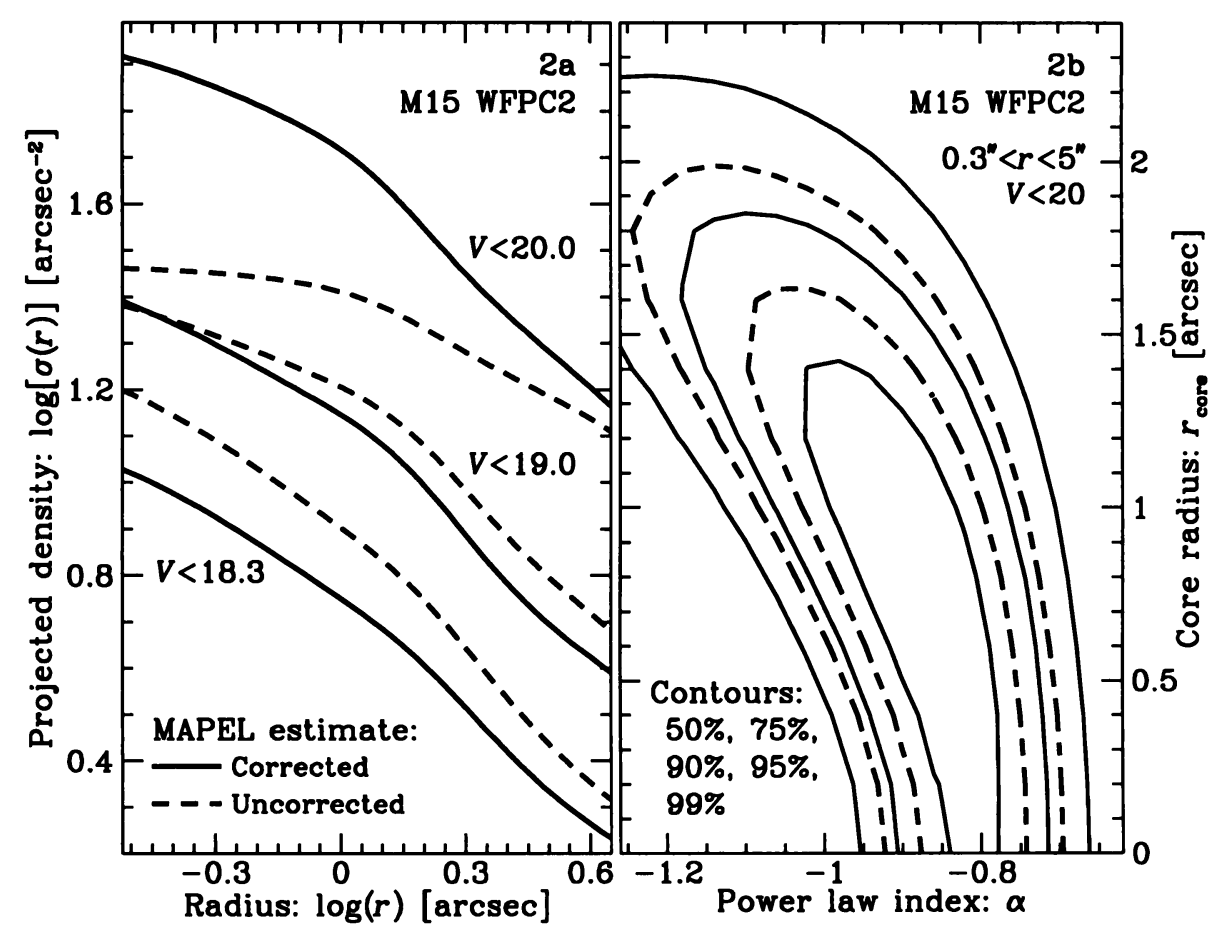

Figure 2. Left (a) Non-parametric (MAPEL) estimate of the stellar surface density profile in the central part of M15 based on HST WFPC2 data. The raw counts (dotted lines) are converted to the corrected profile (solid line) using correction factors derived from a simulation. The different stellar samples yield corrected profiles that are similar in shape. Right (b) The joint probability distribution of the core radius and asymptotic power law index $\alpha$ of M15. The tilt of the likelihood contours indicates significant covariance between the two parameters. The data are consistent with a simple power law of index $\alpha \sim-0.8\left(r_{\text {core }}=0\right)$.

the surface brightness. Magnitude-limited star counts must be corrected for the effects of incompleteness and photometric error which depend strongly on the degree of crowding, which in turn varies with distance from the cluster center. These corrections are determined using detailed simulations of the data for each cluster; each simulation mimics the PSF shape, stellar luminosity function, and surface density distribution of stars in the cluster.

\subsection{M15'S CENTRAL CUSP}

M15 has a strongly peaked surface brightness profile and the highest central density of known globular clusters. These have long made it a promising post core collapse candidate (King 1975) or the site of a central massive black hole (Bahcall et al. 1975). Lauer et al. (1991) suggested that the dif- 
fuse residual light in a pre-repair HST image of M15 has a $2^{\prime \prime}$ core, but realistic simulations of their data indicated that the diffuse light is not a reliable measure of the stellar density because photometric errors lead to inaccurate subtraction of the light of bright giants and because of the complicated nature of the aberrated PSF (Yanny et al. 1994a).

Short WFPC2 $V$ (F555W) exposures of M15 have been used to derive counts of stars above the turnoff. Within $r \lessgtr 3^{\prime \prime}$, crowding is severe despite HST's 0 "' 1 resolution (Fig. 1$)$. The brightest stars $(V<16)$ are easiest to count but their small number leads to large Poisson error in the density estimate. Photometric scatter and bias cause overcount in subgiant samples $(V<18)$ which lie in a particularly steep part of the stellar luminosity function. Fainter samples $(V<20)$ suffer primarily from incompleteness near the cluster center. We have experimented with various limiting magnitudes and independent stellar samples, using the simulation to correct for the above effects in each case, and they all yield consistent results for density profile of M15 indicating that the corrections are reliable (Fig. 2a).

The corrected surface density profile of post main sequence stars in M15 $(V \lesssim 19)$ is well approximated by a power law, $\sigma(r) \propto r^{\alpha}$, with an index $\alpha=-0.82 \pm 0.12$ over the radial range 0 ". $3 \lesssim r \lesssim 6^{\prime \prime}$ (Fig. 2a). Interior to 0.3 , uncertainties in the cluster centroid position, star count correction factor, and star counts (Poisson error) prevent reliable determination of the density. M15's density profile steepens to a slope of $\alpha \sim-1.3$ for $6^{\prime \prime}<$ $r<30^{\prime \prime}$ and to $\alpha \sim-2$ beyond $r=30^{\prime \prime}$. A non-parametric estimate of the spatial density profile of M15 (using the MAPEL method of Merritt and Tremblay 1994) suggests that the density of evolved $V<19$ stars alone exceeds $10^{5} \mathrm{pc}^{-3}$ in the inner $0.02 \mathrm{pc}$.

A power law is an adequate description of the density profile in M15's central $6^{\prime \prime}$. The best fit $\alpha \sim-0.8$ index is consistent with that expected from models of core collapse (cf. Grabhorn et al. 1992) or with the predicted shape of the stellar concentration around a massive black hole (Bahcall and Wolf 1976,1977$)$. While the density profile shows no hint of flattening at small radii, a small but finite core cannot be ruled out by the data. The $99 \%$ and $90 \%$ upper limits to the core radius of M15 are $2 " .2$ and $1 " .8$ (0.1 pc or $0.03 r_{\text {half-mass }}$ ), respectively (Fig. $2 \mathrm{~b}$ ). This is comparable to the core sizes expected from binary heating and core oscillations (Grabhorn et al. 1992).

\subsection{RADIAL DENSITY PROFILES OF OTHER CLUSTERS}

Short HST WFPC2 exposures have been used to study the central regions of the clusters M30 and NGC 6624 (Yanny et al. 1994b, 1996) in a manner similar to the M15 study described above. The density profiles of these two clusters are also well approximated by a single power law in their central 
$5^{\prime \prime}(\sim 0.2 \mathrm{pc})$. The cusp in M30 is somewhat shallower than the one in M15 $(\alpha=-0.4 \pm 0.15)$ while that in NGC 6624 is comparably steep $(\alpha \sim-0.85)$. The central stellar surface densities of both clusters are somewhat lower than that of M15. The groundbased study of Djorgovski and King (1986) placed all three clusters in the 'post core collapse' category, characterized by surface brightness profiles rising towards the center (to the $\sim 1^{\prime \prime}$ resolution limit). As in the case of M15, WFPC2 star counts in M30 and NGC 6624 yield reliable estimates of the density profile down to $r \sim 0$ !' 3 , and these show no hint of flattening into a core. The upper limit on the core radius of the two clusters is comparable to that of M15, roughly $2^{\prime \prime}$ or $0.1 \mathrm{pc}$.

Not every cluster in our sample is of the post core collapse variety. Prerepair WF/PC-I images of the 'King model' clusters M13, M3, and 47 Tuc have been/are being analyzed. All three have relaxed distributions with flat cores of radius roughly $30^{\prime \prime}(\sim 1 \mathrm{pc})$ and very high core stellar densities. Note, the radial distribution of evolved stars in 47 Tuc (measured relative to the number weighted cluster centroid or 'center of mass') is well fit by a King profile with $r_{\text {core }}=23^{\prime \prime} \pm 2^{\prime \prime}(0.5 \mathrm{pc})$ in disagreement with the $8^{\prime \prime}$ core radius found by Calzetti et al. (1993) for ultraviolet selected stars. The lack of any detectable cusp places a $95 \%$ upper limit on the mass of a compact central object in 47 Tuc of about $2500 M_{\odot}$ (Guhathakurta et al. 1992).

\section{Stellar Populations}

The superb angular resolution of HST makes it possible to carry out accurate photometry of faint stars in crowded regions ( $1 \sigma$ color errors $\lesssim$ $0.1 \mathrm{mag}$ ). This is essential in order to reliably define the various stellar types (e.g. red giant, horizontal branch, subgiant, and turnoff stars). The color-magnitude diagrams of the central regions of 47 Tuc and M15 presented in Fig. 3 demonstrate the power of HST. These data are ideal for studying blue straggler stars, in particular, since this population is subject to contamination due to the blending of turnoff stars and inaccurate photometry of subgiants/blue horizontal branch stars in low resolution images.

\subsection{BLUE STRAGGLERS}

The 47 Tuc data were obtained with the pre-repair HST's Planetary Camera and were reduced and analyzed in collaboration with R. Gilliland and P. Edmonds (STScI). The $U$ data consist of a series of $991000 \mathrm{sec}$ exposures which were combined into a very deep, doubly oversampled image ( 1 pixel $=0$ !.023). These data yield a clean and complete sample of BSS out to $r=2 r_{\text {core }}$ in 47 Tuc (see Fig. 3a), extending the samples found by Paresce et al. (1991) and Guhathakurta et al. (1992) in earlier pre-repair HST studies. 


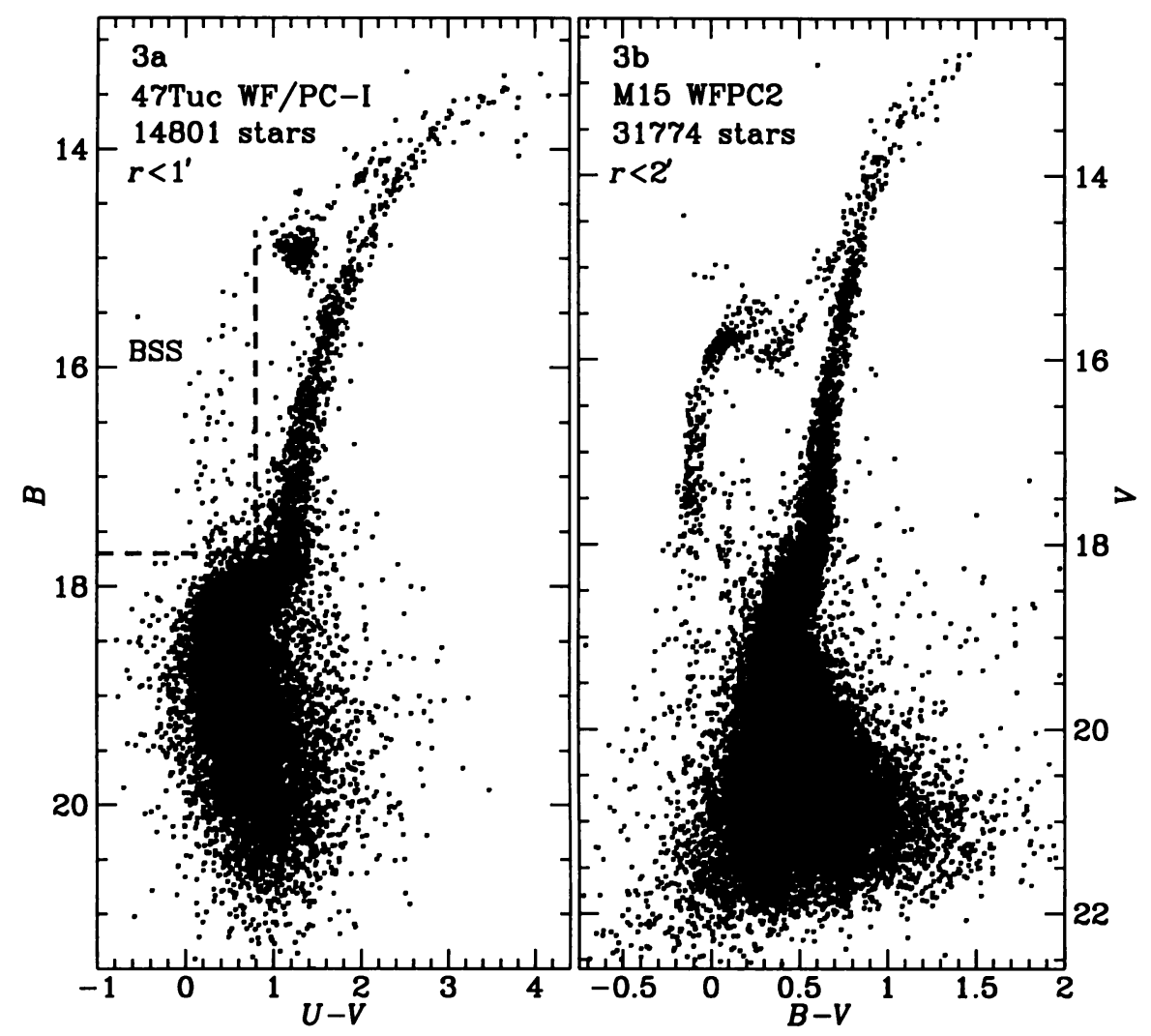

Figure 3. Left (a) A $B$ vs. $U-V$ color-magnitude diagram of the core of 47 Tuc derived from very deep pre-repair HST WF/PC-I data (in collaboration with Gilliland and Edmonds). Note the prominent sequence of blue straggler stars (BSS) extending above the main sequence turnoff. Right (b) A $V$ vs. $B-V$ color-magnitude diagram of the central part of M15 from HST WFPC2 stellar photometry. A small number of blue straggler candidates are visible slightly brighter and bluer than the main sequence turnoff (and to the red of the drooping tail of the blue horizontal branch). Over $3 \times 10^{5}$ stars are detected in the $5 \operatorname{arcmin}^{2}$ area of the WFPC2 mosaic.

Our HST WFPC2 data on M15 reveal a small number of BSS candidates, located 1-2 mag brighter and 0.1-0.4 mag bluer than the main sequence turnoff: $V_{\mathrm{TO}} \approx 19,(B-V)_{\mathrm{TO}} \approx 0.4$ (Fig. $3 \mathrm{~b}$ ). The region of M15's color-magnitude diagram bounded by the drooping blue horizontal branch, subgiants, and turnoff is relatively free of objects. The numerous "yellow stragglers" seen in Stetson's (1994) groundbased data were probably artifacts of blending (as the author suspected!).

We measure the specific frequency of BSS $F_{\mathrm{BSS}}$ (as defined by Bolte et al. 1993 ) in the central regions of several globular clusters: M30, NGC 6624, 
M15, 47 Tuc, M13, and M3. Sosin and King (1995) point out that it is interesting that the scatter in BSS frequency from cluster to cluster is only about a factor of three, with a mean value of $F_{\mathrm{BSS}} \sim 0.12$, despite their $z 10^{4}$ range in central stellar density. Within each of the above clusters though, the BSS are centrally concentrated relative to other evolved stars, with $F_{\mathrm{BSS}}$ increasing by about a factor of 2 between $r \sim 2 \mathrm{pc}$ and the center. This can be explained in terms of mass segregation: BSS in dense cluster cores are thought to be products of stellar collisions with $M_{\mathrm{BSS}} \sim 2 M_{\text {turnoff }}$. There are suggestions of a bimodal BSS population in M3 where $F_{\mathrm{BSS}}$ seems to go through a minimum at $r \sim 2^{\prime}$ or $5 r_{\text {core }}$ (Ferraro et al. 1993). However, our HST WF/PC-I study of the central 1' of M3 (Guhathakurta et al. 1994) shows that the BSS sample derived from groundbased images of this crowded region suffers from the effects of blending and incompleteness, even though the images were obtained in 0".5 seeing (Bolte et al. 1993). Clearly, a uniformly high quality dataset (in terms of angular resolution and depth) is needed, covering the full radial range of the cluster, before definite conclusions can be drawn about the nature of the BSS in M3.

The observed number of BSS relative to other evolved stars serves as a diagnostic for theories of BSS formation. Observational studies of these stars in dense globular cluster cores are beginning to explore areas beyond that probed by BSS counts alone. These include accurate monitoring to study photometric variability caused by SX Phe-type pulsations and eclipsing binaries (cf. Edmonds et al. 1996) and comparison of the BSS luminosity function and color distribution to models of BSS formation and evolution (Bailyn and Pinsonneault 1995).

\subsection{CENTRAL RED GIANT DEFICIENCY IN M15}

Several post core collapse globular clusters display color gradients near their centers indicating changes in the mix of stellar populations in regions of very high density (Piotto et al. 1988; Cederbloom et al. 1992). These gradients are usually in the sense of the overall cluster light becoming bluer inwards and are attributed to a deficiency of the brightest RGB stars. Stetson (1994) convincingly demonstrated this RGB deficiency for the cluster M15. While the brightest giants are relatively easy to detect, even in these very dense regions, it is important to define a complete sample of fainter stars (e.g. subgiants) that can be used as the normalizing population relative to which the RGB deficiency is measured.

We use WFPC2 stellar photometry in the central 100" of M15 (see Fig. $3 \mathrm{~b}$ ) to study the radial variation of the relative numbers of bright RGB stars (defined to be those brighter than the horizontal branch or $V<16)$, horizontal branch stars, and subgiants $(16<V<17.5)$. The 




Figure 4. Radial gradients in the mix of evolved stars near M15's center derived from HST WFPC2 photometry. The fraction of bright red giant branch stars relative to subgiants (solid line) is about a factor of 2 lower in the inner 10" compared to the region further out $\left(r=10^{\prime \prime}-100^{\prime \prime}\right)$. The fraction of horizontal branch stars (dashed line) is roughly constant over the entire $r<100^{\prime \prime}$ region; the $20 \%$ decrease in the inner $10^{\prime \prime}$ and the $50 \%$ increase beyond $r=1^{\prime}$ are not statistically significant ( $\$ 2 \sigma$ effects). There are comparable numbers of bright RGB stars and HB stars in the $r=10^{\prime \prime}-100^{\prime \prime}$ region. Error bars show Poisson errors.

results are shown in Fig. 4. The fraction of bright RGB stars relative to subgiants appears to decrease with decreasing radius inside $r<10^{\prime \prime}$ or $0.6 \mathrm{pc}$ ( $23 \sigma$ significance). The RGB fraction in the inner $10^{\prime \prime}$ is about half the value measured further out. The horizontal branch stars on the other hand exhibit no strong radial variation relative to subgiants. The apparent $20 \%$ decrease in the horizontal branch fraction in the inner $10^{\prime \prime}$ is only a $1 \sigma$ effect and the possible increase beyond $r=1^{\prime}$ a $2 \sigma$ effect; it is worth noting that both red and blue horizontal branch stars display these trends. The central RGB depletion and the constancy of the horizontal branch fraction is independent of the exact definition of the subgiant samplei.e. the results are identical relative to faint or bright subgiant samples. We avoid using turnoff or main sequence stars $(V \gtrsim 18)$ as the normalizing population since counts of these stars are affected by crowding near M15's center (Guhathakurta et al. 1996b).

There are roughly equal numbers of bright RGB and horizontal branch stars in M15's $r>10^{\prime \prime}$ region (Fig. 4), suggesting that a star spends comparable amounts of time in these two evolutionary phases. The fact that 
bright RGB stars are depleted by a factor of two in the dense central $0.6 \mathrm{pc}$ whereas stars on the horizontal branch (evolutionary descendants of RGB stars) are not indicates that the bright RGB phase is 'short circuited' in this region. If the RGB depletion were caused by destruction of giants, we would expect to see a similar central dip in the horizontal branch fraction, and this is not observed.

P.G. would like to thank his collaborators Peter Edmonds and Ron Gilliland for allowing him to present data from an unpublished study of 47 Tuc, the conference organizers for an enjoyable and fruitful week in Tokyo, and the editors for their patience in the months since.

\section{References}

Aurière, M. and Cordoni, J.-P. (1981) A. Ap. S. 46, 347.

Bahcall, J.N., Bahcall, N.A. and Weistrop, D. (1975) Astrophys. Lett. 16, 159.

Bahcall, J.N. and Wolf, R.A. (1976) Ap. J. 209, 214.

Bahcall, J.N. and Wolf, R.A. (1977) Ap. J. 216, 883.

Bailyn, C.D. and Pinsonneault, M.H. (1995) Ap. J. 439, 705.

Bolte, M., Hesser, J.E. and Stetson, P.B. (1993) Ap. J. 408, L89.

Calzetti, D., de Marchi, G., Paresce, F. and Shara, M. (1993) Ap. J. 402, L1.

Cederbllom, S.E., Moss, M.J., Cohn, H.N., Lugger, P.M., Bailyn, C.D., Grindlay, J.E. and McClure, R.D. (1992) A. J. 103, 480.

Cohen, R.L., Guhathakurta, P., Yanny, B., Bahcall, J.N. and Schneider, D.P. (1996) A. J. in prep.

Djorgovski, S. and King, I.R. (1986) Ap. J. 305, L61.

Edmonds, P.D., Gilliland, R.L., Guhathakurta, P., Petro, L.D., Saha, A. and Shara, M.M. (1996) $A$. $J$. in prep.

Ferraro, F.R., Fusi Pecci, F., Cacciari, C., Corsi, C., Buonanno, R., Fahlman, G.G. and Richer, H.B. (1993) A. J. 106, 2324.

Grabhorn, R.P., Cohn, H.N., Lugger, P.M. and Murphy, B.W. (1992) Ap. J. 392, 86.

Guhathakurta, P., Webster, Z., Yanny, B., Bahcall, J.N. and Schneider, D.P. (1996a) $A$. $J$. in prep.

Guhathakurta, P., Yanny, B., Bahcall, J.N. and Schneider, D.P. (1994) A. J. 108, 1786.

Guhathakurta, P., Yanny, B., Schneider, D.P. and Bahcall, J.N. (1992) A. J. 104, 1790.

Guhathakurta, P., Yanny, B., Schneider, D.P. and Bahcall, J.N. (1996b) A. J. 111, 267.

King, I.R. (1975) in Dynamics of Stellar Systems, IAU Symposium No. 69, ed. A. Hayli, Reidel, Dordrecht, p. 99.

Lauer, T.R. et al. (1991) Ap. J. 369, L45.

Merritt, D. and Tremblay, B. (1994) A. J. 108, 514.

Paresce, F. et al. (1991) Nature 352, 297.

Piotto, G., King, I.R., and Djorgovski, S. (1988) A. J. 96, 1918.

Sosin, C. and King, I.R. (1995) A. J. 109, 639.

Stetson, P.B. (1994) P. A. S. P. 106, 250.

Yanny, B., Guhathakurta, P., Bahcall, J.N. and Schneider, D.P. (1994a) A. J. 107, 1745.

Yanny, B., Guhathakurta, P., Schneider, D.P. and Bahcall, J.N. (1994b) Ap. J. 435, L39.

Yanny, B., Guhathakurta, P., Schneider, D.P. and Bahcall, J.N. (1996) A. J. in prep. 